\title{
Electrophoretic Tissue Clearing and Labeling Methods for Volume Imaging of Whole Organs
}

\author{
Dai Hyun Kim ${ }^{1}$, Hyo Hyun Ahn ${ }^{2}$, Woong Sun ${ }^{1,3}$, Im Joo Rhyu ${ }^{1,3, *}$ \\ ${ }^{1}$ Department of Anatomy, Korea University College of Medicine, Seoul 02841, Korea \\ ${ }^{2}$ Department of Dermatology, Korea University College of Medicine, Seoul 02841, Korea \\ ${ }^{3}$ Division of Brain Korea 21 Plus Program for Biomedical Science, Korea University College of Medicine, Seoul 02841, Korea
}

*Correspondence to:

Rhyu IJ,

Tel: $+82-2-2286-1150$

Fax: +82-2-2286-1387

E-mail: irhyu@korea.ac.kr

Received September 6, 2016

Revised September 21, 2016

Accepted September 21, 2016

\begin{abstract}
Detailed structural and molecular imaging of intact organs has incurred academic interest because the associated technique is expected to provide innovative information for biological investigation and pathological diagnosis. The conventional methods for volume imaging include reconstruction of images obtained from serially sectioned tissues. This approach requires intense manual work which involves inevitable uncertainty and much time to assemble the whole image of a target organ. Recently, effective tissue clearing techniques including CLARITY and ACT-PRESTO have been reported that enables visualization of molecularly labeled structures within intact organs in three dimensions. The central principle of the methods is transformation of intact tissue into an optically transpicuous and macromolecule permeable state without loss of intrinsic structural integrity. The rapidly evolving protocols enable morphological analysis and molecular labeling of normal and pathological characteristics in large assembled biological systems with single-cell resolution. The deep tissue volume imaging will provide fundamental information about mutual interaction among adjacent structures such as connectivity of neural circuits; meso-connectome and clinically significant structural alterations according to pathologic mechanisms or treatment procedures.
\end{abstract}

Key Words: Volume imaging, Tissue clearing technique, Single-cell resolution, Morphological analysis, Molecular labeling

\section{INTRODUCTION}

The volume imaging of intact organs/tissues with singlecell resolution provides fundamental understanding of 3-dimensional (3D) organization of biological specimens. It will also improve our knowledge about generation and progression of diseases with chronic debilitating process such as neurodegenerative diseases or cancers, which is certainly helpful to the development of next generation diagnostic or treatment methods (Renier et al., 2014; Tainaka et al., 2014). The conventional approaches for volume imaging require physical section of biological tissues and subsequent multiple processing of images, because the technically accessible depth for acquiring tissue information (e.g., structure, protein distribution, etc.) is limited within hundreds of micrometers primarily due to the diffusion limit of the macromolecule probes (e.g., antibody) and the light scattering (Helmchen \& Denk, 2005; Tang et al., 2012; Tomer et al., 2014). Therefore, the conventional approaches for volume imaging are laborintensive, time-consuming, requiring specialized apparatus, and essentially error-prone (Gong et al., 2013; Kim et al., 2014; Li et al., 2010).

The recent advent of tissue clearing and labeling methods have been substantially resolved the above-mentioned obstacles through selective removal of lipid component in target tissues or organs without loss of the original protein

(a) This is an open-access article distributed under the terms of the Creative Commons Attribution Non-Commercial License (http://creativecommons.org/licenses/by-nc/4.0) which permits unrestricted noncommercial use, distribution, and reproduction in any medium, provided the original work is properly cited.

Copyrights @ 2016 by Korean Society of Microscopy 
structures, and/or the use of optical solution with proper adjustment of refractive index (RI) (Chung et al., 2013; Hama et al., 2011; Ke et al., 2013; Tomer et al., 2014). The resulted biological specimens showed elevated optical transparency and permeability of macromolecules including labeling reagent. Therefore, direct 3D imaging with single cell resolution becomes feasible without mechanical sections. The lipid components are electively extracted either by passive diffusion (Renier et al., 2014; Susaki et al., 2014) or actively by electrophoresis (Chung et al., 2013; Lee et al., 2016a; Tomer et al., 2014).

The electrophoretic methods have been conducting a leading role in the growing field of tissue clearing since this breakthrough technique was firstly reported in 2013 (Fig. 1). In this review, we will focus on the recently reported electrophoretic lipid removal method combined with effective labeling technique which enables rapid clearing and effective immuno-labeling (Lee et al., 2016a).

\section{HYDROGEL-BASED TISSUE CLEARING PROCEDURES}

The tissue clearing techniques shared similar principles in the way that extracting lipids in a stable hydrophilic chemical compounds. The CLARITY is one of the well-known methodologies applying hydrophilic hydrogel monomers (HMs) such as acrylamide with or without bisacylamide (Chung et al., 2013; Tomer et al., 2014). The CLARITY constructs fundamental chemical basis to build supporting

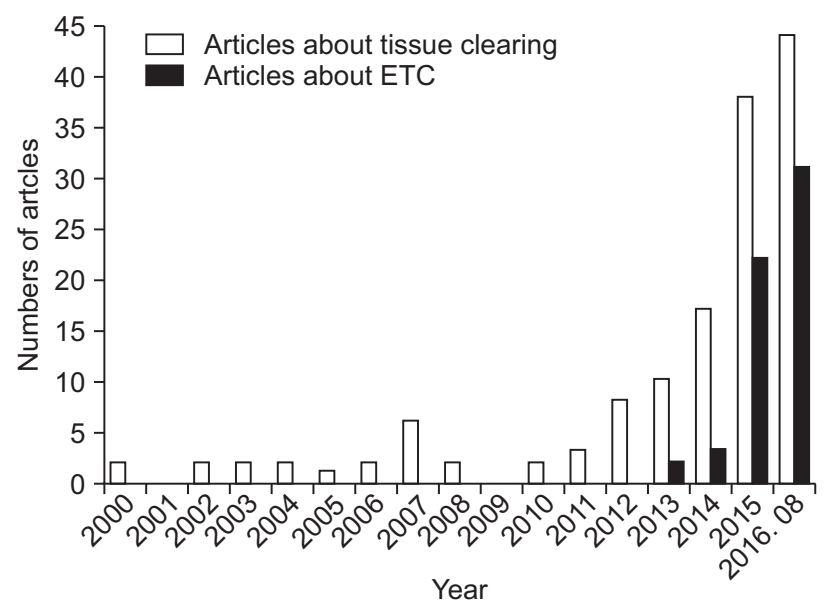

Fig. 1. The annually categorized graph showed noticeable elevation of reported articles about tissue clearing techniques in recent years. The electrophoretic tissue clearing (ETC) method was emerged in 2013 and has been positioning in leading role to the significant growth. All of the articles in the graph was searched in PubMed with separate and/or combinative input of keywords including 'Tissue clearing, 'BABB', 'ClearT', 'Sca/e,' '3DISCO,' 'CLARITY,' 'SeeDB,' 'CUBIC, 'PACT/PARS,, 'iDISCO, 'Sca/ eS, 'SWITCH, 'ACT-PRESTO', and 'EDC-CLARITY'. framework through infusion of HMs from surface to the deep portion of target tissues to sustain structural and biomolecular integrity during the clearing process. The active clarity technique (ACT) is the advanced tissue clearing method which enables more shortened duration of clearing compared with CLARITY (Lee et al., 2016a).

The ACT protocols are composed of four steps: 1) tissue fixation, 2) HM embedding, 3) polymerization, and 4) electrophoretic tissue clearing (ETC) (Fig. 2). The paraformaldehyde tissue fixation is proceeded and the subsequent step is infusing the mixture of HM, acylamide without bis-acrylamide, and thermal initiator, VA-044, under cold environment $\left(4^{\circ} \mathrm{C}\right)$. The polymerization is initiated under controlled condition with $37^{\circ} \mathrm{C},-70 \mathrm{kPa}$ and vacuum state. The HMs undergo active covalent binding with native biomolecules including proteins, nucleic acids except vast majority of cellular membranous phospholipids. The degree of protein-acrylamide crosslinking is less formidable in ACT compared with CLARITY and the resulted higher porosity hydrogel facilitates more rapid tissue clearing and labeling (Chung et al., 2013; Lee et al., 2016a). The unbounded lipid components are then extracted without disintegration of original biomolecular organization of target tissues or organs through ionic detergent-based clearing solution [boratebuffered $4 \%$ sodium dodecyl sulfate (SDS)] at $37^{\circ} \mathrm{C}$ with active electrophoretic forcing. The effective lipid removing process aided by electrophoresis is called ETC.

The ETC originally reported in CLARITY protocol showed several limitations such as long duration of clearing process with more than 1 2 weeks in case of whole mouse brain and potential risk of tissue damage because of unstable temperature, uncontrolled changes in $\mathrm{pH}$ and blocking of circulatory current by air bubbles (Lee et al., 2016a; Tomer et al., 2014). The improved ETC chamber reported in ACT system is equipped with platinum plate to achieve dense and consistent current around a target tissue. In addition, the quality of ETC buffer solution is sustained with relatively coherent color and $\mathrm{pH}$ during prolonged duration of lipid extraction and active cooling device in the ACT system prevents significant changes of temperature. Furthermore, comparably tall ETC chamber allows all air bubbles float to the above and discharged through the top aperture (Fig. 3). Therefore, ACT-ETC enables whole organ clearing within $4 \sim 20$ hours without significant damages to the clarified tissues (Lee et al., 2016a). The speed of tissue clearing is essential factor for some applications such as $3 \mathrm{D}$ tissue diagnosis, and thus ACT would be suitable for these applications.

\section{CLEARING DENSE AND EXTRACELLULAR MATRIX-RICH ORGAN}

Tissue clearing and labeling methods have been noticeably 
A

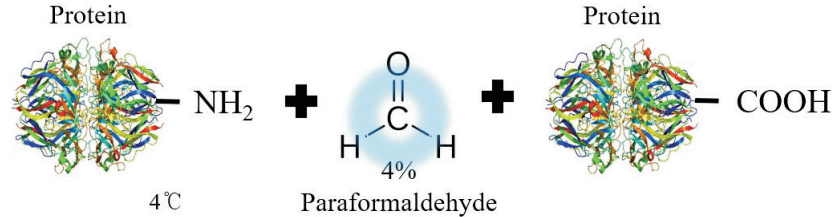

B

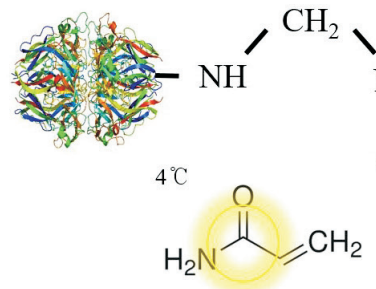

Acylamide
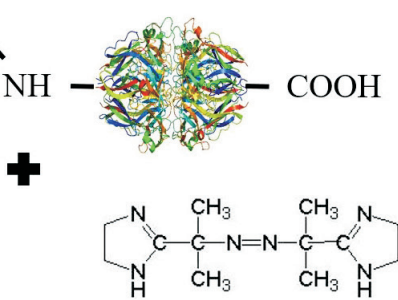

Thermal initiator
$2 \mathrm{HCl}$

C
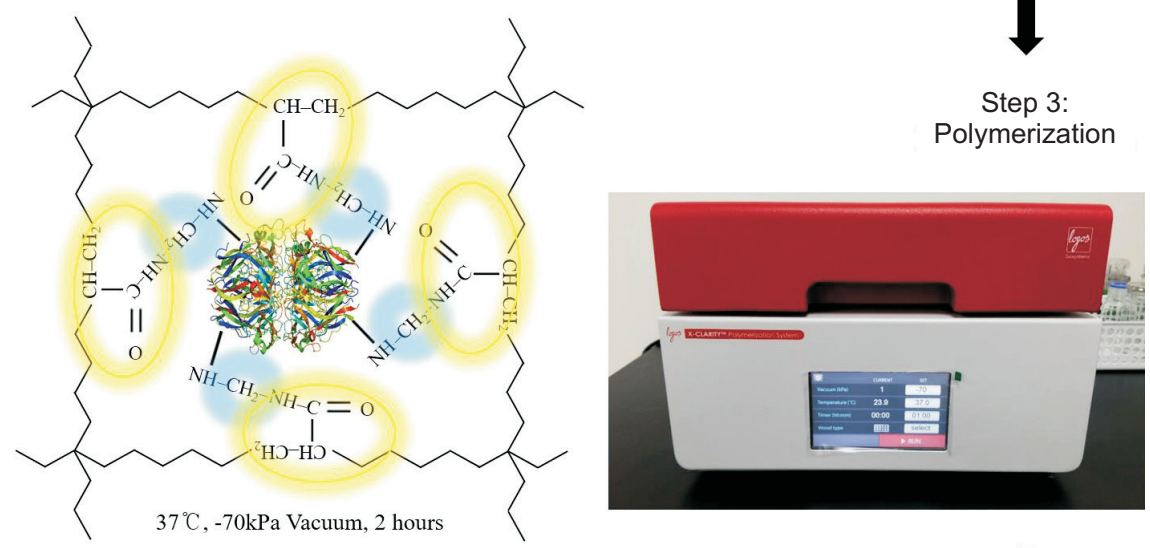

D
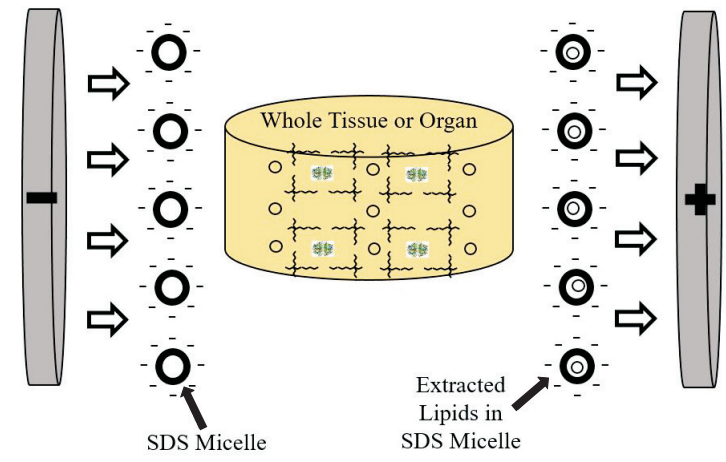

Step 1:

Tissue fixation

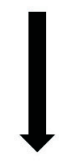

Step 2:

Hydrogel

embedding

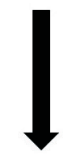

Step 3:

Polymerization

Step 4: Electrophoretic tissue clearing
Fig. 2. The schematic workflow of hydrogel-based electrophoretic tissue clearing procedures composed of 4 steps: tissue fixation, hydrogel monomer infusion, polymerization, and electrophoretic tissue clearing. (A) The proteins in target tissues are fixed with $4 \%$ paraformaldehyde and (B) immersed in a $4 \%$ acrylamide solution admixed with thermal initiator, VA-044. (C) The whole tissue is polymerized in the device with controlled environments at $37.0^{\circ} \mathrm{C},-70$ $\mathrm{kPa}$ and vacuum state for 2 hours. (D) Lastly, the lipid membranes comparably free from the cross-linking are selectively removed from the tissue by electrophoresis. improved with the appearance of innovative device and reagents. The majority of clearing protocols provide satisfactory results with soft tissues such as brain tissues (Chung et al., 2013; Lee et al., 2016a; Susaki et al., 2014; Tomer et al., 2014). However, ETC dependent lipid removal is not sufficient for clearing of dense and extracellular matrix (ECM)-rich organs such as liver, kidney, and skin to produce the near complete transparency achieved in clarified brain (Fig. 4A). The partially cleared ECM rich organs through ETC steps could be additionally cleared through immersion in the refractive index matching solution (RIMS) which reduced light scattering in the immerged tissue (Lee et al., 2016; Tomer et al., 2014; Yang et al., 2014). The RIMS embedding triggers diminished RI variations among heterogeneous components in tissues, mounting media and lens of an imaging tool. The appropriate values of RI for complete tissue clearing have been reported as 1.38 1.56 (Lee et al., 2016a; Seo et al., 2016; Yang et al., 2014). 


\section{LABELING METHODS FOR EFFECTIVE PENETRATION OF MACROMOLECULE INTO DENSE TISSUES}

The effective labeling method is crucial for obtaining structural and contextual biological information from

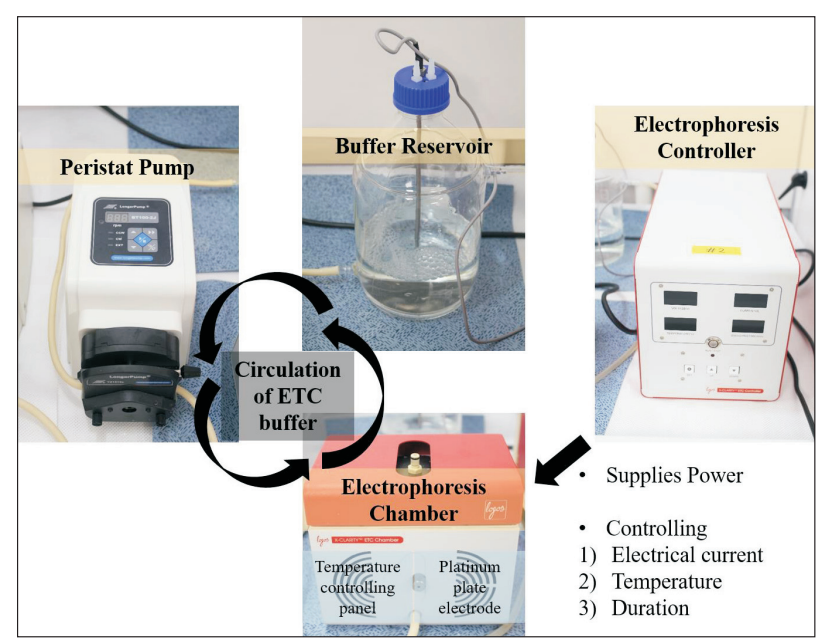

Fig. 3. Detailed processes of the active clarity technique-electrophoretic tissue clearing (ACT-ETC). The whole tissue is placed in the electrophoresis chamber. The electrophoresis controller supplies regular power to the chamber and contributes to generate regular electrical current and sustain controlled temperature in the chamber also accomplished by temperature controlling panels and platinum plate electrodes. The peristat pump provides continuous flow of sodium dodecyl sulfate buffer and allows effective removal of lipids from the tissue as well as air bubbles generated in the chamber. the thick tissues. The traditional labeling methodologies including immunohistochemistry (IHC) depend on passive diffusion of specific antibodies and therefore involve intrinsic limitations by slow diffusion rate and shallow penetrable depth. Several latent articles reported the solutions facilitating the transportation of macromolecular probes into the thick and dense tissues (Chung et al., 2013; Kim et al., 2015; Lee et al., 2016a). The related techniques are categorized into 3 groups: 1) elevation of permeability of target specimen, 2) administering external forces and 3) regulating the kinetics of probe-target binding reaction. First, specific macromolecules can be easily penetrate into the target tissue by hydrogelbased tissue clearing protocols including ETC as mentioned above, because this procedure removes one of the major physical barrier, lipid. In addition, this method also results mild expansion of the tissues which also contribute to the enhanced diffusibility of the macromolecules. Second, macromolecular transportation is effectively accelerated through applying controlled pressure generated by centrifuge or syringe pump to the tissue and this method is called c-PRESTO and s-PRESTO respectively (Lee et al., 2016a). The PRESTO methods especially provide effective and rapid penetration of labeling reagent into the dense and ECM rich organs including liver and kidneys (Fig. 4B and C). Another approach using external forces utilizes controlled electrical field and facilitates the movement of electrically charged molecules. Stochastic electrotransport efficiently expanded the differences between comparably high and low electromobile molecules (Kim et al., 2015). The free molecules have greater electromobilities compared with cross-linked
A

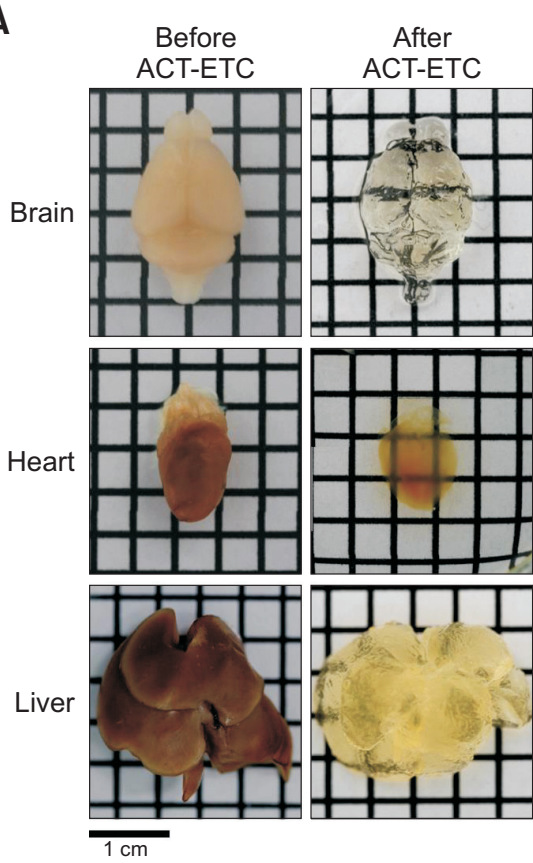

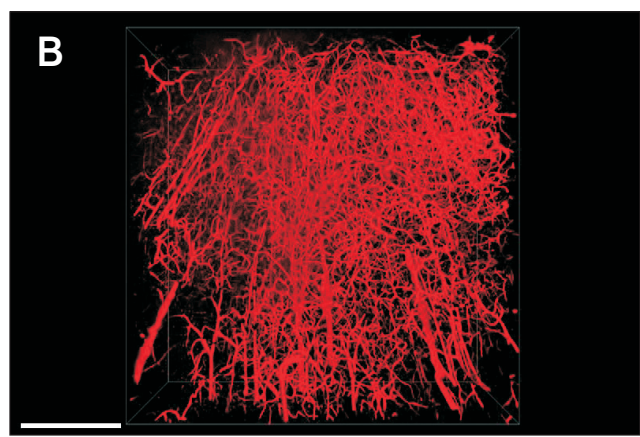

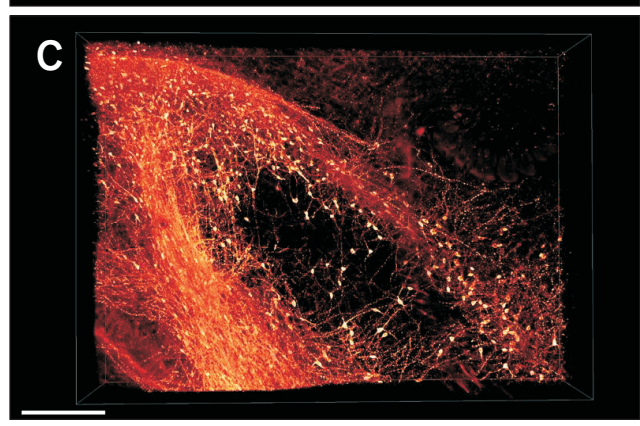

Fig. 4. The results of whole organ clearing by the active clarity technique (ACT) and immunolabeling. (A) The degree of clearing by ACT-electrophoretic tissue clearing (ETC) could be varied according to the composition of target tissues. The mouse brain and heart showed complete or near complete clearing however, the opposite findings are obtained with the liver, extracellular matrix rich organ. Scale bar=1 cm. (B) The integral structures of blood vessels in clarified brain are labeled with type IV collagen aided with c-PRESTO technique. (C) The distribution and connectivity of dopaminergic neurons can be visualized by labeling with tyrosine hydroxylase. (B, C) All three-dimensional reconstructed images were obtained by confocal miscroscope (Leica SP8, Leica Microsystems $\mathrm{GmbH}$, Germany) with a Plan-Apochromat $10 \times$ lens. Scale bar $=500$ $\mu \mathrm{m}$. 
endogenous protein of target tissue. Therefore, antibodies are readily penetrated while sustaining the original organization of protein structures (Kim et al., 2015; Seo et al., 2016). Third, the method termed SWITCH improves the penetration of macromolecules into the tissues through modulating kinetics of binding reactions between probes and specific proteins in target tissues (Murray et al., 2015). SWITCH protocols uses two kinds of buffers called SWITCH-Off and SWITCHOn buffers. The labeling probe with high concentration can disperse throughout the entire target tissue without nonspecific binding in the SWITCH-Off buffer. The SWITCHOn buffer subsequently activates the binding and therefore enables both improved penetration depth and uniformed labeling (Murray et al., 2015).

\section{APPLICATIONS OF ELECTROPHORETIC TISSUE CLEARING AND LABELING METHODS IN BASIC RESEARCH}

The reported articles about tissue clearing and labeling techniques have been remarkably increased in recent years and the emergence of electrophoresis-assisted method, CLARITY, ignites the significant growth of basic research using clarified tissue (Fig. 1). All of the tissue clearing methods are invented for acquisition and correlating molecular and structural information even in large whole-organs with high resolution across diverse length scales. Therefore, most studies with existing or advanced clearing and labeling method have been applied to the molecular phenotyping of a variety of animal, human whole organs and even plants (Kurihara et al., 2015; Palmer et al., 2015).

The clearing and labeling methods provide fluorescence imaging of certain proteins and mRNA in intact, whole organs (Lee et al., 2016b). Animal or human brains have been the most frequently applied organs of clearing techniques and therefore, the meso-scaled neural connectivity, i.e., meso-connectomes, in brains have been being the highly concentrated topics in this field (Chung et al., 2013; Renier et al., 2014; Tomer et al., 2014; Yang et al., 2014). Dopamenergic neurons and their projections are reported to be immunostained with tyrosine hydroxylase in brains (Fig. 4C) and dorsal root ganglia (Renier et al., 2014). In addition, the transgenic mice expressing fluorescence proteins allow efficient means to visualize specific subset of neural substrates in cleared brains. For instance, cholinergic neurons and associated projections could be visualized under the control of choline acetyl transferase and the excitatory neuronal projections could be imaged with Thyl::GFP or YFP lines (Chung et al., 2013; Renier et al., 2014; Tomer et al., 2014). The familiar non-neuronal structures such as blood vessels and tubular structures are reported to be labeled within cleared brains and/or peripheral organs (Chung et al., 2013; Lee et al., 2016a). Further investigative categories related with tissue clearing and labeling are evaluation of cellular proliferation using BrdU, EdU or phosphohistone H3, assessment of neuronal activity with immediate early genes including c-fos and research about apoptosis and neurodegeneration through caspase-3 (Renier et al., 2014).

\section{CLINICAL IMPLICATIONS OF ELECTROPHORETIC TISSUE CLEARING AND LABELING METHODS}

Only small number of articles reported the clearing and labeling results of human tissues and the specimens were post-mortem human brain or spinal cord (Chung et al., 2013; Lee et al., 2016a; Murray et al., 2015). The tissue clearing and labeling methods were successfully able to achieve volume imaging of neurons and projections in brains of both normal and autistic patients (Chung et al., 2013). The cleared brains showed preserved neurite continuity and provide the topographical information about 3D distribution of neurons in particular layer of brain. Therefore, the clearing and labeling techniques are anticipated to contribute to reveal complex pathophysiological mechanism of neuropsychiatric disease and sleep disorders (Chung et al., 2013; Kanda et al., 2016). For example, the clarified human brain samples with mutations in the Down syndrome cell adhesion molecule protein or protocadherin could show that many parvalbuminpositive interneurons in deep layers have unusual forms of dendritic bridges compared with the neurons in normal brain (Chung et al., 2013). In addition, the 3D structures of amyloid plaques found in Alzheimer's disease (AD) could be imaged through clearing techniques and the results are anticipated to be utilized in modifying the current classification system and advanced knowledges about initial cause of $\mathrm{AD}$ (Liebmann et al., 2016). Lastly, the proteomic imaging abilities of SWITCH could evaluate the distances between different types of cells in brain and vessels within a single intact tissue. Therefore, the studies are able to be designed about structural relationships between cerebral vasculature and brain cells using this method (Murray et al., 2015).

The human tissues or organs obtained from patients are the potentially promising specimens which could be the appropriate target for tissue clearing and labeling. The recently developed ETC and labeling methods are not only able to overcome the limitations of IHC, the major tool for confirmative diagnosis in clinical field but also allow rapid acquisition of massive but accurate volumetric images. Therefore, the biopsied and sectioned tissues from patients for diagnostic and/or therapeutic purposes are the important sources that could create and broaden the clinical implications of this promising technology. 


\section{CONCLUSIONS}

The ETC and labeling methods are the effective techniques to obtain reliable and rapid clearing of thick and dense tissues. The methods can also label deep structures of particular organs or even a whole organisms. Therefore, volume imaging of intact tissues with single-cell resolution can be obtained within a few days without labor-intensive and time-consuming section-reconstitution procedures. These techniques enable the acquisition of detailed 3D inter- relationships among hundreds of millions of cells as well as different structural tissue components. Both basic and clinical investigations will contribute to evolve the technologies and find adoptable diagnostic and/or therapeutic implications in near future.

\section{CONFLICT OF INTEREST}

No potential conflict of interest relevant to this article was reported.

\section{REFERENCES}

Chung K, Wallace J, Kim S Y, Kalyanasundaram S, Andalman A S, Davidson T J, Mirzabekov J J, Zalocusky K A, Mattis J, Denisin A K, Pak S, Bernstein H, Ramakrishnan C, Grosenick L, Gradinaru V, and Deisseroth K (2013) Structural and molecular interrogation of intact biological systems. Nature 497, 332-337.

Gong H, Zeng S, Yan C, Lv X, Yang Z, Xu T, Feng Z, Ding W, Qi X, Li A, Wu J, and Luo $Q$ (2013) Continuously tracing brain-wide long distance axonal projections in mice at a one-micron voxel resolution. Neuroimage $\mathbf{7 4}$, 87-98.

Hama H, Kurokawa H, Kawano H, Ando R, Shimogori T, Noda H, Fukami K, Sakaue-Sawano A, and Miyawaki A (2011) Scale: a chemical approach for fluorescence imaging and reconstruction of transparent mouse brain. Nat. Neurosci. 14, 1481-1488.

Helmchen F and Denk W (2005) Deep tissue two-photon microscopy. Nat. Methods 12, 932-940.

Kanda T, Tsujino N, Kuramoto E, Koyama Y, Susaki E A, Chikahisa S, and Funato $\mathrm{H}$ (2016) Sleep as a biological problem: an overview of frontiers in sleep research. J. Physiol. Sci. 66, 1-13.

Kim S Y, Cho J H, Murray E, Bakh N, Choi H, Ohn K, Ruelas L, Hubbert A, McCue M, Vassallo S L, Keller P J, and Chung K (2015) Stochastic electrotransport selectively enhances the transport of highly electromobile molecules. Proc. Natl. Acad. Sci. U S A 112, E6274-E6283.

Ke M T, Fujimoto S, and Imai T (2013) SeeDB: a simple and morphologypreserving optical clearing agent for neuronal circuit reconstruction. Nat. Neurosci. 16, 1154-1161.

Kim H W, Kim D S, and Rhyu I J (2014) Electron tomography and synapse study. Appl. Microsc. 44, 83-87.

Kurihara D, Mizuta Y, Sato Y, and Higashiyama T (2015) ClaerSee: a rapid optical clearing reagent for whole-plant fluorescence imaging. Dev. Camb. Engl. 142, 4168-4179.

Lee E, Choi J, Jo Y, Kim J Y, Jang Y J, Lee H M, Kim S Y, Lee H J, Cho K, Jung N, Hur E M, Jeong S J, Moon C, Choe Y, Rhyu I J, Kim H, and Sun W (2016a) ACT-PRESTO: rapid and consistent tissue clearing and labeling method for 3-dimensional (3D) imaging. Sci. Rep. 6, 18631.

Lee E, Kim H J, and Sun W (2016b) See-through technology for biological tissue: 3-dimensional visualization of macromolecules. Int. Neurourol. J. 20, S15-S22.
Li A, Gong H, Zhang B, Wang Q, Yan C, Wu J, Liu Q, Zeng S, and Luo Q (2010) Micro-optical sectioning tomography to obtain a highresolution atlas of the mouse brain. Science 330, 1404-1408.

Liebmann T, Renier N, Bettayeb K, Greengard P, Tessier-Lavigne M, and Flajolet M (2016) Three-dimensional study of Alzheimer's disease hallmarks using the iDISCO clearing method. Cell Rep. 26, 11381152.

Murray E, Cho J H, Goodwin D, Ku T, Swaney J, Kim S Y, Choi H, Park Y G, Park J Y, Hubbert A, McCue M, Vassallo S, Bakh N, Frosch M P, Wedeen V J, Seung H S, and Chung K (2015) Simple, scalable proteomic imaging for high-dimensional profiling of intact systems. Cell 163, 1500-1514.

Palmer W M, Martin A P, Flynn J R, Reed S L, White R G, Furbank R T, and Grof C P L (2015) PEA-CLARITY: 3D molecular imaging of whole plant organs. Sci. Rep. 5, 13492.

Renier N, Wu Z, Simon D J, Yang J, Ariel P, and Tessier-Lavigne M (2014) iDISCO: a simple, rapid method to immunolabel large tissue samples for volume imaging. Cell 159, 896-910.

Seo J, Choe M, and Kim S Y (2016) Clearing and labeling techniques for large-scale biological tissues. Mol. Cells 39, 439-446.

Susaki E A, Tainaka K, Perrin D, Kishino F, Tawara T, Watanabe T M, Yokoyama C, Onoe H, Eguchi M, Yamaguchi S, Abe T, Kiyonari H, Shimizu Y, Miyawaki A, Yokota H, and Ueda H R (2014) Whole-brain imaging with single-cell resolution using chemical cocktails and computational analysis. Cell 157, 726-739.

Tainaka K, Kubota S I, Suyama T Q, Susaki E A, Perrin D, Ukai-Tadenuma M, Ukai H, and Ueda H R (2014) Whole-body imaging with single-cell resolution by tissue decolorization. Cell 159, 911-924.

Tang J, Germain R N, and Cui M (2012) Superpenetration optical microscopy by iterative multiphoton adaptive compensation technique. Proc. Natl. Acad. Sci. U S A 109, 8434-8439.

Tomer R, Ye L, Hsueh B, and Deisseroth K (2014) Advanced CLARITY for rapid and high-resolution imaging of intact tissues. Nat. Protoc. 9, 1682-1697.

Yang B, Treweek J B, Kulkarni R P, Deverman B E, Chen C K, Lubeck E, Shah S, Cai L, and Gradinaru V (2014) Single-cell phenotyping within transparent intact tissue through whole-body clearing. Cell 158, 945958. 